\title{
Sensing motion using spectral and spatial analysis of WLAN RSSI
}

\author{
Kavitha Muthukrishnan, Maria Lijding, Nirvana Meratnia and Paul Havinga \\ University of Twente, Faculty of Computer Science \\ Computer Architecture Design and Test for Embedded Systems group \\ P.O.Box 217, 7500 AE, Enschede, The Netherlands \\ \{k.muthukrishnan, m.e.m.lijding, n.meratnia, p.j.m.havinga\}@ewi.utwente.nl
}

\begin{abstract}
In this paper we present how motion sensing can be obtained just by observing the WLAN radio signal strength and its fluctuations. The temporal, spectral and spatial characteristics of WLAN signal are analyzed. Our analysis confirms our claim that 'signal strength from access points appear to jump around more vigorously when the device is moving compared to when it is still and the number of detectable access points vary considerably while the user is on the move'. Using this observation, we present a novel motion detection algorithm, Spectrally Spread Motion Detection (SpecSMD) based on the spectral analysis of WLAN signal's RSSI. To benchmark the proposed algorithm, we used Spatially Spread Motion Detection (SpatSMD), which is inspired by the recent work of Sohn et al. Both algorithms were evaluated by carrying out extensive measurements in a diverse set of conditions (indoors in different buildings and outdoors - city center, parking lot, university campus etc.,) and tested against the same data sets. The $94 \%$ average classification accuracy of the proposed SpecSMD is outperforming the accuracy of SpatSMD (accuracy 87\%). The motion detection algorithms presented in this paper provide ubiquitous methods for deriving the state of the user. The algorithms can be implemented and run on a commodity device with WLAN capability without the need of any additional hardware support.
\end{abstract}

\section{Introduction}

Ubiquitous computing is emerging as an exciting new paradigm with a goal to provide services anytime anywhere. Context is a critical parameter of ubiquitous computing. The ubiquitous computing applications make use of several technologies to infer different types of user context. The context cue that we are interested in is users motion being either 'moving' or 'still'. Knowledge about whether a user or device is still or in motion can serve a number of purposes. The mobility status is an important part of a users context and is thus of interest to context-aware systems, e.g. [13]. Knowing the users motion is very useful for activity recognition and localization [11].

It is possible to use specialized sensors and beacons, such as accelerometer, pedometer or motion sensor [2] to measure certain aspects of users motion. Although they offer precise motion inference, they are too expensive and obtrusive for wide deployments. Specialised motion tracking and motion detection systems make use of a range 
of sensing technologies [14]. Although such dedicated systems typically provide highly accurate information concerning position and even orientation they require additional hardware, which is often unwieldy, impractical or simply not available. Hence, solutions which do not require a dedicated hardware for determining the state of the user are gaining popularity [3][1][8][9]. Particularly, there is a growing interest in using the existing infrastructure and technology to derive motion status.

In the last few years, reading emails and surfing the internet whilst on the move at public places, has become an accepted part of daily life with WLAN standard being integrated into laptop, handhelds and cellular phones. Location based services, using WiFi tags in settings like hospitals to track equipments and patients are currently the trend [13], [12] and localization using WiFi [12] [5] [7] is considered as a value added application to WiFi networks. In this paper, we provide-Motion inference as a useful mechanism which adds incremental value to WiFi networks. The main advantages of the proposed motion detection are: (i) deducing motion of the user using the existing infrastructure $(\mathrm{WiFi})$ without a need of additional hardware, as it offers a pure software based solution (ii) preserving user privacy, as motion inference is performed locally at the client device.

\subsection{Motivation}

The motion detection algorithms that we present in this paper provide ubiquitous methods for deriving the state of the user. The algorithms can be implemented and run on a commodity device with WLAN capability without the need of any additional hardware support. The recognition of device/users motion status will lead to a number of applications, including personalized assistants , smart monitoring and surveillance systems, as well as motion analysis in sports and medical domain. In the section below we envision three use cases of the motion detection algorithm presented in this paper.

A. Enhancing WLAN localization accuracy Applying the motion information to WLAN localization algorithm may result in accuracy improvement in the existing Wireless LAN positioning systems, such as RADAR [5], Flavour [7] and Ekahau [12]. These systems essentially use received signal strength typically measured at the mobile device to estimate location. In general, signal samples fluctuate even in environments with few moving objects. Thus resulting in an unstable location estimation even while the user or the device is still. In order to overcome errors due to instability in RSSI used for location estimation, most positioning algorithms use some kind of time averaging to smoothen the RSSI values . Inspite of time averaging the instability still exists, resulting in lesser accuracy. The performance and hence accuracy of signal strength-based localization can be improved if knowledge about the user state,i.e. moving or still is incorporated as a part of localization algorithm itself. As an example, when the user state is deduced as still, all the unwanted jumps caused by the location estimation can be ignored. When the user state is deduced as moving, an appropriate motion model can be added as a part of the location algorithm. Therefore after each location update the new location estimate can move by a maximum distance depending on user's speed. In this way, many false location estimations can be curtailed, leading to better accuracy. 
B. Healthy living at Office Office workers tend to lead a very sedentary life, thereby making them very prone to various diseases like Repetitive Strain Injury (RSI). Recently health centers such as, Rehabilitation Center Het Roessingh (RRD) [15] is promoting healthy living in the office environment with a help of providing personalized assistance, which determines the user's activity level and use it to give timely feedback, warnings or advice to the users. For instance, if the user is continuously sitting for a long time, the system can give feedback or encourage the user to move, if the user is already moving, it can keep a count of how much the person has moved and so forth. With just a mobile handheld, an employee would be able to gather information about his daily activity level and get appropriate feedbacks. Motion recognition forms vital part of such applications, by improving the condition of the employee at work.

C. Activity recognition An impressive amount of research falls under the umbrella of activity recognition. Be it for elderly care support or for social coordination [8], motion detection forms an important part. The WLAN radio by itself can sense motion, and it can potentially be also part of the sensor ensemble to improve recognition performance. Adding machine learning algorithms might result in identifying more states, thus making it very useful for fine-grained activity recognition.

The applications that are described above do not necessarily benefit from accurate and complete information about the mobility status. For the purposes described above it is sufficient to know whether the user is moving or not. However, all the above mentioned applications requires reliability- low false alarm rate, low latency-inferring motion status without much delay, instantaneously at the best and light computation-to efficiently run on mobile hand held devices.

\subsection{Contributions and Outline}

The key contributions of our work are:

1. For the first time, the spectral characteristics of WLAN signals RSSI are analyzed. Our analysis results in a conclusion that "when a device is moving, signal strengths of all heard access points vary much greater and more obvious compared to when a device is still and the number of detectable access points vary considerably when the device is moving". This analysis is presented in Section 3.2.

2. We propose a novel motion detection algorithm called Spectrally Spread Motion Detection (SpecSMD). In this algorithm, the WLAN signal in time series is transformed to frequency domain by employing Fast Fourier Transform. By analyzing the spectral width of the fourier transformed signal, a two-state classification scheme is used to deduce if a user is moving or still. We discuss the algorithm in Section 4.1. The experimental results are outperforming the existing motion detection algorithms in use today by achieving an overall classification accuracy of approximately $94.1 \%$. The evaluation of SpecSMD is addressed in Section 5.2.

3. Additionally, we present Spatially Spread Motion Detection (SpatSMD) which is inspired by [8]. We discuss it in Section 4.2. SpatSMD achieves an average classification accuracy of $86.7 \%$ in deducing the correct motion status. 
Section 2 presents the related work. We outline the data collection process used in gathering the WLAN signal traces in Section 5.1. Section 6 concludes the paper and presents ongoing work.

\section{Related work}

It is possible to infer the state of the user by the usage of external hardware such as an accelerometer. Randell et al. [2] demonstrated the possibility of distinguishing various states of the movement such as walking, climbing and running using a $2 \mathrm{D}$ accelerometer. Patterson et al. [9] take the velocity readings from GPS measurements and infer the transportation mode of the user, for instance walking, driving, or taking a bus using a learning model. The model learns the traveler's current mode of transportation as well as his most likely route, in an unsupervised manner. It is implemented using particle filters and is learned using Expectation-Maximization. The learned model can predict mode transitions, such as boarding a bus at one location and disembarking at another. The research now is focusing on finding a methodology to obtain the same information using the existing infrastructure or capability of the device.

A promising alternative to usage of specialized hardware is to investigate what can be obtained by measuring signal received from existing infrastructures (either WLAN or GSM). Since WiFi access points and WiFi clients are ubiquitous, this technique is very attractive. In this line, Krumm et al. [3] classified a user as either moving or still based on the variance of a temporally short history of signal strength from currently the strongest access point. This classification had many transitions, hence it was smoothened over the time with a two-state hidden markov model (HMM) resulting in an overall accuracy of $87 \%$. Privacy is enhanced compared to systems that compute context on a central server, since the context inferences rely only on client-side data and computations.

Anderson et al. [1] use GSM cellular signal strength levels and neighboring cell information to distinguish movement status. The classification of the signal patterns is performed using a neural network model resulted in an average classification accuracy of $80 \%$. The authors trained the neural network initially and demonstrated a proof of concept by implementing it at run time on a cell phone. However the initial training did not work in all the environments as signal strength fluctuations were different in different environments.

Recently Sohn et al. [8] published a similar technique for detecting users motion using signal traces from GSM network. Their motion detection system yields an overall accuracy of $85 \%$. They extracted a set of 7 features to classify the user state as either still, walking, or driving.

\section{$3 \quad 802.11$ radio channel characteristics}

In this section, we begin with a description of our scanning process used to log WLAN signal readings for studying the radio channel characteristics. We then discuss the RF signal propagation and the noisy wireless channel characteristics. Subsequently we analyze the temporal, spectral and spatial characteristics of RSSI of the WLAN signal 
which forms the basis of the motion detection algorithm presented in this paper. To study the signal propagation and perform measurements, we used a HP IPAQ pocketPC with built-in WLAN card. We used a Spotter [6] [7] to capture the RSSI from each access point.

\subsection{Scanning Process}

The IEEE 802.11 standard defines a mechanism by which RF energy is to be measured by the circuitry on a wireless NIC. In $802.11 \mathrm{~b} / \mathrm{g} / \mathrm{a}$, this numeric value is an integer with an allowable range of $0-|255|$ and called as the Received Signal Strength Indicator (RSSI). The IEEE 802.11 does not require that a chipset vendor use all 255 values, so each vendor will have a specific maximum value. For example, Cisco chooses RSSImax as 100 while the atheros chipset use 60 as the maximum value. Thus, the Spotter [6] [7] measures a signal strength value between 0 and $|255|$ for each of the access points in the vicinity. Figure 1(B) shows an output example of Spotter. The spotter outputs a list of the MAC addresses of access points accompanied with the time-stamped signal strength observed in the scan (probe response frames). Any WLAN device using the Spotter can scan for nearby access points. Additionally the user can log which activity he was performing at the time of measurement and specify where the measurement was made (see Figure 1(A)). This phase is typically known as Scanning phase.

Tradeoff between Scanning rate vs battery-life Continuous scanning and logging WLAN access points can expend a devices battery in few hours. While continuous scanning provides rich depiction of a users dynamic environment, most individuals expect their personal devices to have more standby times. Hence, for a longer battery life, the scanning interval should be as large as possible. However, with a very large scanning period, the motion updates are also much less frequent. Therefore we have used spotter to scan the environment once every 4 seconds.

\subsection{Analyzing temporal, spectral and spatial characteristics of WLAN signal}

The IEEE $802.11 \mathrm{~b}$ standard operates in the $2.4 \mathrm{GHz}$, Industrial-Scientific-Medical (ISM) band. Since the ISM band is open to anyone, radio systems operating in this band must cope with several unpredictable sources of interference, such as baby monitors, garage door openers, cordless phones, other radio such as bluetooth and microwave ovens (the strongest source of interference). There are two types of variations associated with the wireless channel [10], -Small scale variations and Large scale variations. Small scale variation are equivalent to the correlated fading experiences in the wireless channel for close sampling distances. This happens when the receiver moves over a very small distance. Handling small scale variations is very challenging. Large scale variation on the other hand describes the characteristics of the channels structure when the distance between the transmitter and the receiver increases considerably. The large scale variation is significant as over long distances the signals get attenuated. The indoor radio signal propagation is often subjected to reflection, refraction, diffraction and absorption by structures and even human bodies, thus signal propagation suffers from severe 


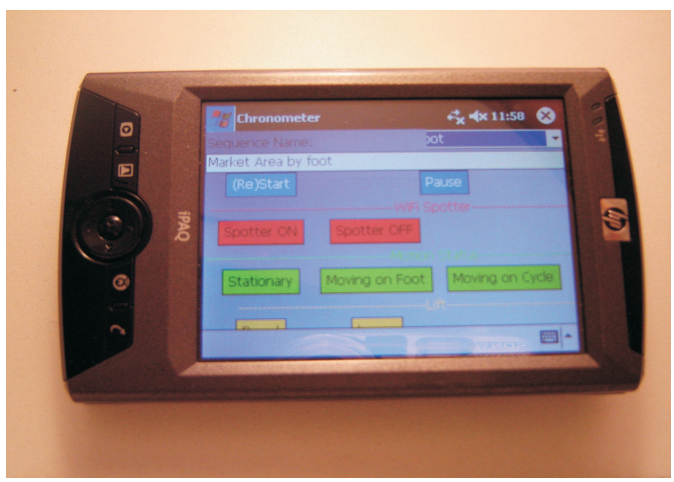

(A)

Measurment Fri May 25 12:14:44 CEST 2007

(B)

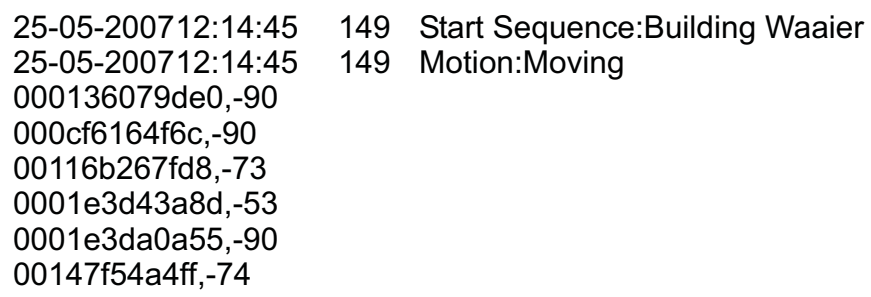

Fig. 1. (A). Screen shot of WiFi Spotter Application, illustrating the data collection diary, (B). An example of Spotter output

multi-path fading effects in an indoor environment [10]. As a result, a transmitted signal can reach the device through different paths, each having its own amplitude and phase. These different components combine and reproduce a distorted version of the original signal. Moreover, changes in the environmental conditions, such as temperature or humidity affect the signals to a large extent.

Temporal Characteristics This subsection describes how the wireless channel changes over time when the user is standing still and moving. Figure 2(A) presents signal strength and its variations over time from all the heard access points. We measured (numerous measurements were performed at different settings (indoors/outdoors) and representative data is used for plotting) the signal strength a little over 2 minute time interval. From the figures it is obvious that even at a fixed position the signal strength varies, however the variation is not more than $10-15 \mathrm{dBm}$. This happens mainly because motion of people or equipments that are present in indoor environments make the radio channel nonstationary in time, that is the channel's statistics change even when the transmitter and receiver are fixed. But when the user is moving (refer Figure 2(B))the 


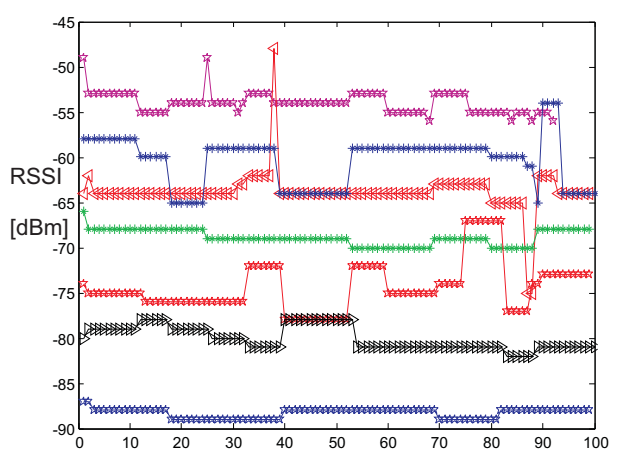

(A)

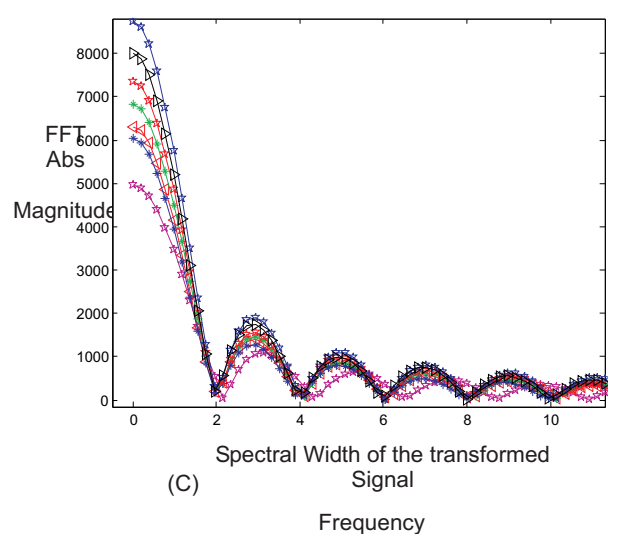

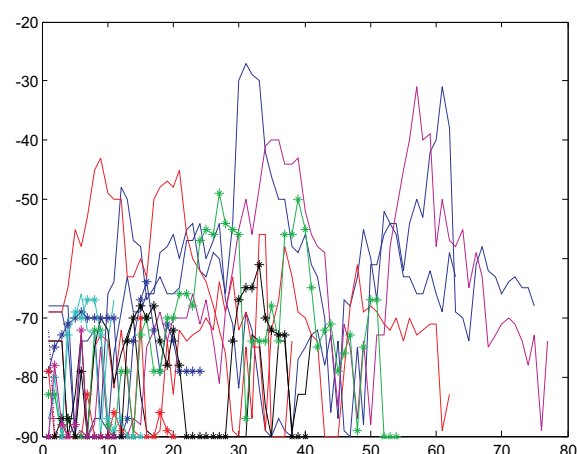

(B)

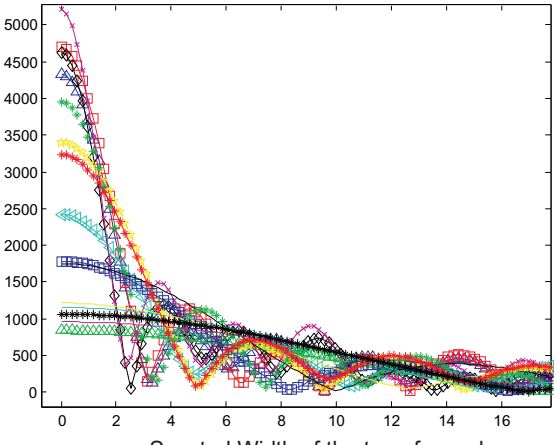

(D)
Signal

Frequency

Fig. 2. (A). Temporal characteristics of signal when user is 'still',(B). Temporal characteristics of signal when user is 'moving',(C). Spectral characteristics of signal when user is 'still', (D). Spectral characteristics of signal when user is 'moving'

signal variations can be as high as $45-60 \mathrm{dBm}$. Hence the variation in signal due to the motion of device has greater influence than the variation due to the dynamics of the changing environment, i.e. motion of people and objects. One can see from the figure that, when the user is still, the fading of the signal occurs as bursts lasting few seconds. However, for moving case the fading of signal is more and persistent. Furthermore, it is clear from Figure 3 that at a fixed location the access points that are detectable do not vary too much, meaning the number of signal strength samples received from the same access point does not vary much. In the case of moving, the number of signal strength samples received from the access point varies as the number of access point detectable at a place varies as the user moves.

Spectral characteristics Analysing the signals spectral characteristics might give a different representation. Hence for converting the WLAN signal in time series to frequency domain we used Fast Fourier Transform (FFT) which enables us to view the frequency representation of the signal. The FFT of $N$ points $\mathrm{x}_{n}$ is defined as follows: 


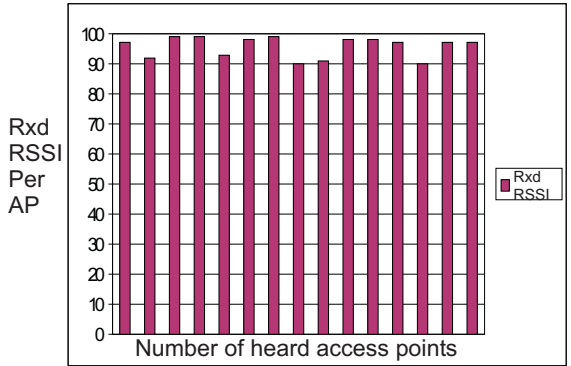

(A)

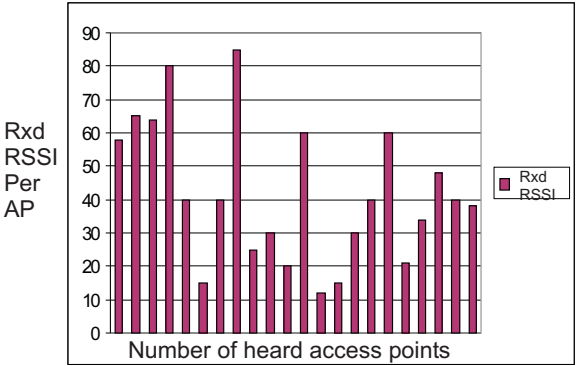

(B)

Fig. 3. Variation in the number of samples received when (A). Still (B). Moving

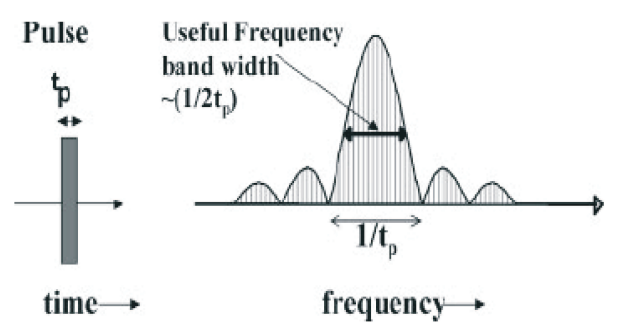

(A)

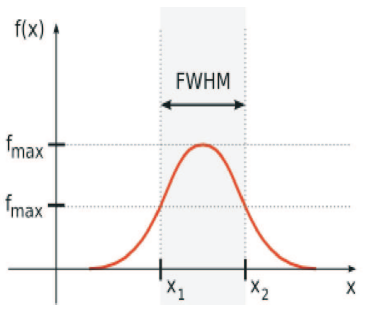

(B)

Fig. 4. (A). Schematic representation of a rectangular pulse in time and frequency domain, short duration pulses produces a large bandwidth,(B). Full Width Half Maximum, corresponding to peak width at $50 \%$ peak height

$$
X_{k}=\sum_{n=0}^{N-1} x_{n} e^{-2 \pi i k n / N} k=0, \ldots . N-1
$$

where $X_{k}$ is the $\mathrm{k}^{\text {th }}$ coefficient of the FFT and $x_{n}$ denotes the $\mathrm{n}^{\text {th }}$ sample of the time series which consists of $N$ samples and $i=\sqrt{-1}$. In our case, $x_{n}$ is the RSSI time series obtained from the Spotter.

The schematic representation of how a signal looks in time and frequency domain is illustrated in Figure 4(A). As a rule of thumb, the more concentrated the time domain, the more spread out the frequency domain. In particular, if we "squeeze" a function in time, it spreads out in frequency and vice-versa. Therefore, a function which equals its Fourier transform strikes a precise balance between being concentrated and being spread out. Also Figure 4(B) illustrates Full Width Half Maximum (FWHM) that corresponds to peak width of the FFT signal at $50 \%$ peak height.

Figure 2(C) \& (D) represent the variation of signal strength from all the heard access points in the frequency domain when the user is still and when the user is moving. It is evident that although signal strength vary even while the user is still, this variation is reflected in all the heard access points uniformly as there is a well defined peak with a narrow spectral width in the frequency domain from all the access points, despite the 
fact that there is difference in the fourier amplitude from each of the heard access point. But when the user is moving, there is no well defined peak from all the access point in the frequency domain indicating that the variation in the signal strength happens more often and not in all the heard access points in the same manner. Furthermore, one can see the effect of spectral broadening from a significant number of access points when the user is moving, resulting in a higher full width half maximum. This phenomenon happens mainly due to two reasons: (i) the variation in the signal strength is large in case of moving user and (ii) number of access points detectable vary with distance resulting in too few received samples from the access points. This confirms that both the temporal and spectral analysis lead to the similar conclusions but give a different view of representation. However the advantage of spectral analysis is that even when the number of samples are few, meaning that the received signals are captured only for an instance of time, it still exhibits the same behavior.

Spatial characteristics Here we present our observation of the signal strength fluctuations reflected in the euclidean space. In particular we want to see: $(i)$ whether any signal correlation for spatially seperated signals exists for the case when user is moving and (ii) how the signal correlates over time for the case when the user is still.

The observation is based on the same principle as fingerprinting-based location systems [5], which state that the signals observed from the access points are consistent in time but variable in euclidean space. Conceptually the Euclidean distance should give a cue on whether a user is moving or still. We conducted experiments to analyze the signal fluctuation and to see how it varies in space. We used Euclidean distance between WLAN measurements as a measure to find out if there is any difference between a moving and a still user. Figure 5(A) illustrates the average Euclidean distance between WLAN measurements and shows that the average Euclidean distance between WLAN measurements are proportional to the state of the movement. When the user is still, the Euclidean distance is relatively small $(<4)$, when the user is moving the Euclidean distance is higher (typically $>4$ ). In a generalized form, the euclidean distance between any two measurements can be written as:

$$
\sqrt{\sum_{i=1}^{n}\left(S_{i(X)}-S_{i(X+C)}\right)^{2}}
$$

where $i$ is the index of the access point used, $X$ is the index of measurement and $C$ is a constant which can vary from 1 till 10 (refer to Section 4.2 for more details on choice of $C$ ).

We estimate rank correlation coefficient using the Spearman Rank Correlation Coefficient [4]. The rank correlation coefficient between any two measurements represent how closely the signals are ranked. It takes the value between 0 and 1 . Ranking closer to 1 indicates that the measurements are similar and hence the user is still and when the user is moving the ranking is close to 0 . Figure 5(B) presents how the rank correlation coefficient behaves when the user is still and moving.

With these observations presented in Section3.2 as the basis, we now present our motion detection algorithms in the next section. 

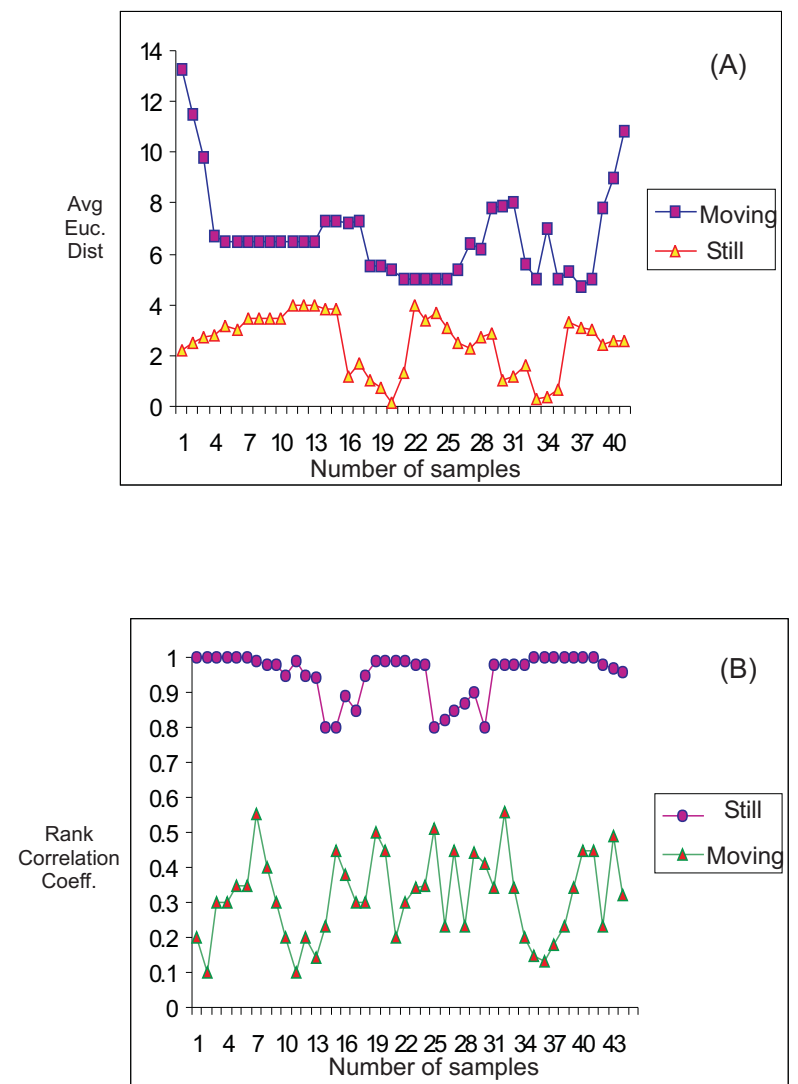

Fig. 5. (A). Spatial characteristics of signal,when user is 'still', and 'moving' (B). Rank correlation Coefficient when the user is still and moving

\section{Motion Detection Algorithms}

\subsection{Spectrally-Spread Mobility Detection (SpecSMD)}

In this subsection we present a novel motion detection algorithm which is based on our observations explained in section 3.2. The algorithm tracks how many access points have spectral width that is exceeding a certain threshold within the window of readings. Intuitively a larger spread indicates that the device is moving.

Algorithm 1 presents the pseudocode of the Spectrally Spread Motion Detection algorithm (SpecSMD). The input to the algorithm is the spotter output comprising of the WLAN readings. The WLAN signal reading in time series is converted into frequency domain using Fast Fourier Transformation (FFT). As a next step, the full width half maximum i.e. peak width of the FFT signal at 50\% peak height is calculated for each of the MAC entries in the spotter output. We are interested in a two-state classifying scheme in which state 1 and state 2 are dealing with deducing still and moving states, respectively. To make a decision based on the full width half maximum, a threshold is set. Whenever a MAC entry exceeds the FWHM threshold, the algorithm treats this as 

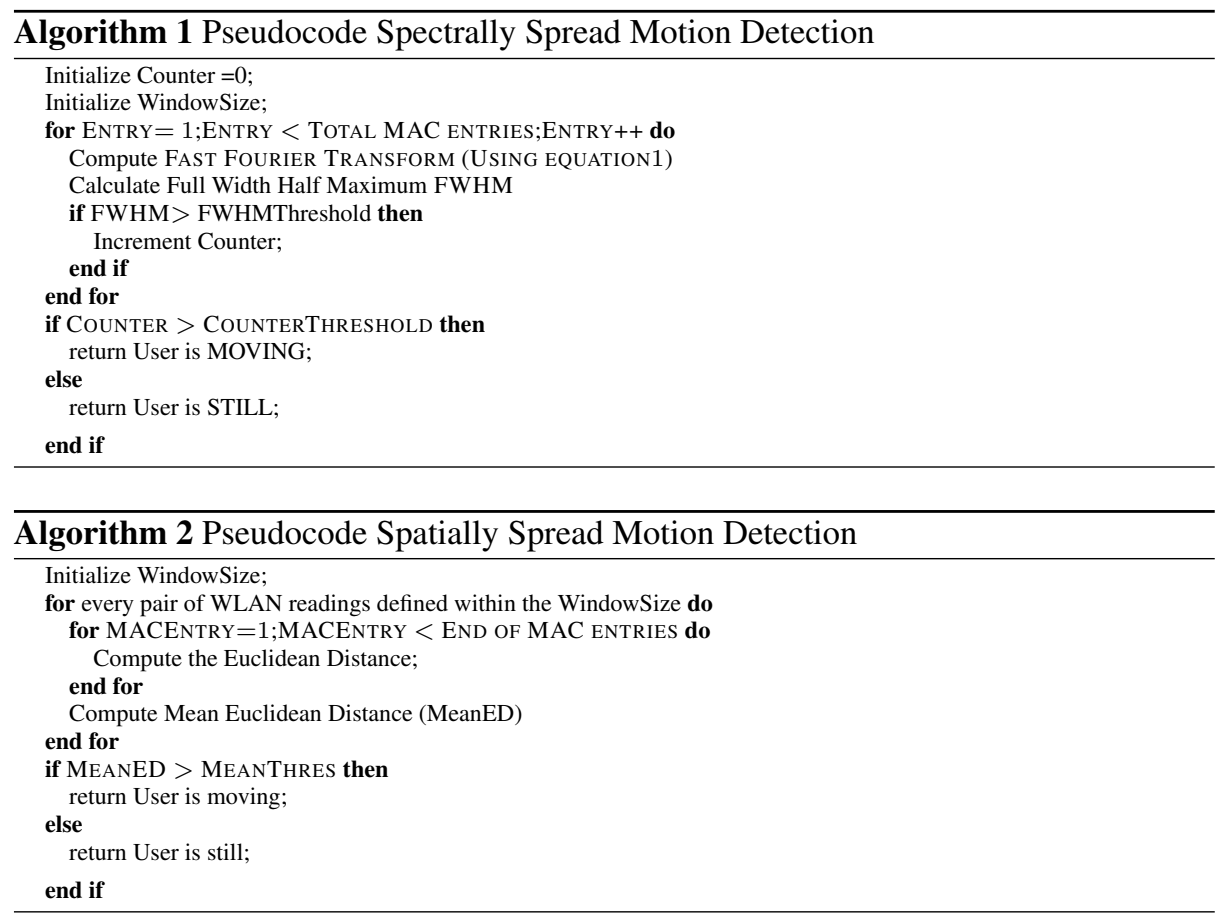

an outlier and increments a counter. If the number of the counter is more than a certain threshold the algorithm returns the user state as Moving, otherwise it returns Still. The number of the counter indicates that many of the access points that are detected have a spectral width exceeding certain threshold. This algorithm is implemented in MATLAB and currently performs offline analysis to classify the state of the user's motion. The fast fourier transformation results in a complexity of $\operatorname{NLog}(\mathrm{N})$, where $N$ refers to the number of samples. We use smaller values of $N$ (we used $\mathrm{N}=6$ ) hence the computation load is negligible.

\subsection{Spatially-Spread Motion Detection Algorithm (SpatSMD)}

The algorithm presented in this subsection is based on our observation explained in section 3.2. We used the spotter to record the connected and the neighboring access points. The algorithm tracks the euclidean distance between the WLAN readings using a sliding window of WLAN readings, as defined by the window size. Intuitively larger euclidean distance indicates that the device is moving. SpatSMD uses Mean euclidean distance as a metric to distinguish between still and moving states. We found that euclidean distance between consecutive measurement does not provide any useful information to infer the state of the user (inspite of lower sampling frequency $0.25 \mathrm{~Hz}$ i.e. compared to $1 \mathrm{~Hz}$ sampling frequency in [8]). In our experiments we found the optimal $C$ value as 5 (refer Section 5 for details). Since we are interested in using only a two state classification (to distinguish between still and moving) it suffices that we 
just use Mean Euclidean distance over a window of measurements, where the values are calculated between measurements and then averaged together. The pseudocode of spatially-spread motion detection algorithm is presented in Algorithm 2.

\section{Experimental evaluation of SpecSMD and SpatSMD}

\subsection{Data collection phase}

Two members of our research team collected WLAN network traces while doing their daily activities for about a week. Each data collector carried a HP IPAQ pocket PC running spotter for recording readings from nearby access points and logging them. Data collectors recorded their mobility activities using a custom diary application running on the PDA that allowed them to indicate whether they were walking, driving, cycling or in one place. Each time a measurement of spotter was logged the associated activity performed at that instance was recorded as ground truth manually (refer Figure 1(A)) from the pull down menu of the Spotter application to make the diary logging more accurate. Data collection was performed at common places such as city center, parking lot, university campus and indoor at the office, canteen and home. In all, the spotter logs contained WLAN traces of different periods ranging from 1 minute to over 15 minutes.

\subsection{Results and Discussion}

In this subsection, we evaluate how accurately the SpecSMD and SpatSMD can differentiate between moving and still states.

SpatSMD Algorithm Out of the logs that were collected during the data collection phase, a representative data, collected from diverse range of settings is plotted in the Figure 6(A). The logs included, data with various access points densities, to see the impact of the number of access point on the results. The least number of access points in the data collected was 4 , including the traces collected at home in several experiments. Figure 6(A) shows the accuracy results of the SpatSMD for $\mathrm{C}=1, \mathrm{C}=5$ and $\mathrm{C}=10$. The higher the value of $\mathrm{C}$, the higher is the lag associated in making the decision. Since we sample once in every 4 seconds, for $C=10$, we need to obtain data for atleast 40 seconds to make the decision. In order to reduce the computation latency, we should keep $\mathrm{C}$ small. Hence we chose $\mathrm{C}=5$ as an optimal value as it performs better than $\mathrm{C}=1$. The data collected from the "Outdoor By Foot" category (refer Figure 6(A)), was captured in dense vegetation area near farms and heard access point was 4 . A reason for the low accuracy could be because of the dense vegetation or could be the number of heard access points. The average classification accuracy obtained with SpatSMD algorithm $(86.7 \%)$ is slightly better than the one reported by Sohn et al. [8](85\%). A reason for the better performance of our algorithm could be the kind of radio technology we are using (i.e. WLAN instead of GSM) and a different sampling frequency $(0.25 \mathrm{~Hz}$ as opposed to $1 \mathrm{~Hz}$ ). However, Sohn et al. achieved this accuracy for a three state classification scheme. Perhaps employing other features as reported in Sohn et al. might result in a significant improvement in the accuracy. This is yet to be investigated. We are yet to investigate the performance of the algorithm under low access point density $(<4)$. 
SpecSMD Algorithm Figure 6(B) shows the accuracy results of the SpecSMD for various values of the FWHM Thresholds and Counter Thresholds. We set the threshold based on a trial-and-error method, by testing the various threshold values on different data sets that were gathered from a wide range of settings. As explained before FWHM refers to full width half maximum i.e. peak width of the FFT signal at $50 \%$ peak height. We collected at least 6 samples, before FFT is computed. Hence the spotter should be $\mathrm{ON}$ at least for 24 seconds to be able to run the motion detection algorithm explained above. This is comparable with SpatSMD (with $\mathrm{C}=5$, it takes approximately the same time).

SpecSMD performs very well for all experimental settings by achieving an overall classification accuracy of $94.1 \%$, clearly outperforming all the existing motion inference algorithms. It is thus reasonable to conclude that the schemes detection accuracy and performance is significant after being tested across various settings. Fine tuning the thresholds might even further increase the accuracy. Figure 6(C) shows the one-toone comparison in the results obtained from both algorithms tested against the same datasets. The performance of SpecSMD (tested on same WLAN traces) is good even in the experiments in which SpatSMD was achieving only 50\% (refer Figure 6(C)). The data collected from the "Outdoor By Foot" category, was taken in a dense vegetation area near farms and the access point density was 4, this is perhaps why the accuracy is low. Nevertheless, the performance of the SpecSMD is better than SpatSMD (78\% against 50\%). The accuracy obtained is much higher than the accuracy reported by Krumm et al. [3] using an algorithm based on the temporal variation of RSSI.

\section{Conclusion and outlook}

The motion detection algorithms presented in this paper provide ubiquitous methods for deriving the state of the user. The algorithms can be implemented and run on a commodity device with WLAN capability without the need of any additional hardware support. Compared to accelerometer, this is a software solution so it could be easily applied to laptops, PDA and cellphones with WiFi capability. Compared to GSM based motion detection, WiFi based motion detection has a different application setting, like for instance improving WLAN positioning accuracy. In this paper for the first time, spectral characteristics of WLAN signals RSSI is analyzed. Based on the analysis we present a novel motion detection algorithm, Spectrally Spread Motion Detection (SpecSMD). We benchmarked the proposed algorithm, against Spatially Spread Motion Detection (SpatSMD). Both algorithms were evaluated by carrying out extensive measurements in a diverse set of settings (both indoors and outdoors). SpecSMD achieved a classification accuracy of $94.1 \%$, outperforming the accuracy of SpatSMD $(86.7 \%)$ and all the existing motion detection in the literature. Although context like moving or still can be deduced from external sensors like accelerometer, the techniques presented in this paper are promising as both these algorithms use only the radio signal that the wireless device should have to perform its normal operation. Both algorithms are reliable, have low latency and are computationally efficient to be able to run on mobile hand helds.

Ongoing work includes implementing both algorithms at run time on a PDA. We are interested in combining the techniques with machine learning algorithms to generate 
more states of classification which is useful for fine grained activity recognition. We are also currently working on incorporating the presented schemes as a part of WLAN localization algorithm.

\section{Acknowledgements}

We thank Andre Kokkler from Computer Architecture for Embedded Systems (CAES) group at the University of Twente for supporting us during temporal and spectral analysis of WLAN signal. This work is part of the Smart Surroundings project, funded by the Ministry of Economic Affairs of the Netherlands under the contract no. 03060.

\section{References}

1. Ian Anderson and Henk Muller, Context Awareness via GSM Signal Strength Fluctuation. In the 4th International Conference on Pervasive Computing, Late breaking results, pages 27-31. Oesterreichische Computer Gesellschaft, May 2006.

2. Cliff Randell and Henk Muller, Context awareness by analysing accelerometer data. In Blair MacIntyre and Bob Iannucci, editors, The Fourth International Symposium on Wearable Computers, pages 175-176, IEEE Computer Society, October 2000.

3. John Krumm and Eric Horvitz, LOCADIO: Inferring Motion and Location from Wi-Fi Signal Strengths, First Annual International Conference on Mobile and Ubiquitous Systems: Networking and Services (Mobiquitous 2004), pages 4-13, August 2004.

4. Weisstein, Eric W, "Spearman Rank Correlation Coefficient." From MathWorld-A Wolfram Web Resource. http://mathworld.wolfram.com/

5. P. Bahl and V. N. Padmanabhan, Radar: An in-building rf-based user location and tracking system, In Proceedings of the IEEE Infocom 2000, Tel-Aviv, Israel, vol. 2, pages 775-784, March 2000, http://citeseer.ist.psu.edu/bahl00radar.html

6. Anthony LaMarca and Yatin Chawathe and Sunny Consolvo and Jeffrey Hightower and Ian Smith and James Scott and Tim Sohn and James Howard and Jeff Hughes and Fred Potter and Jason Tabert and Pauline Powledge and Gaetano Borriello and Bill Schilit, Place Lab: Device Positioning Using Radio Beacons in the Wild, Proceedings of Pervasive'05, Munich, Germany, pages 225-242, May 2005.

7. Kavitha Muthukrishnan, Nirvana Meratnia,Maria Lijding, Georgi Koprinkov and Paul Havinga, WLAN location sharing through a privacy observant architecture, 1st International Conference on Communication System Software and Middleware (COMSWARE), published by IEEE Communication Society Press, Los Alamitos, California, January 2006, paper no 43.

8. Timothy Sohn, Alex Varshavsky, Anthony LaMarca, Mike Y. Chen, Tanzeem Choudhury, Ian Smith, Sunny Consolvo, Jeffrey Hightower, William G. Griswold, and Eyal de Lara, Mobility Detection Using Everyday GSM Traces, Proceedings of the Eighth International Conference on Ubiquitous Computing. Irvine, California, pages 212-224, September 2006.

9. Lin Liao, Donald J. Patterson, Dieter Fox, Henry Kautz, Inferring High-Level Behavior from Low-Level Sensors, In Proceedings of Fifth International Conference on Ubiquitous Computing, UBICOMP2003, Seattle, WA, pages 73-89, October 2003.

10. Homayoun Hashemi, The Indoor Radio Propagation Channel, In Proceedings of IEEE, Volume 81, No 7, July 1993.

11. Jeffrey Hightower, The Location Stack, Phd Thesis, University of Washington,2004.

12. Exahau Positioning System, http://www.ekahau.com. 
13. Air Location II, http://www.engadget.com/2006/10/02/hitachis-employee-trackingairlocation-ii-tag-w-wifi-enabled-rf/

14. GregWelch and Eric Foxlin. Motion tracking: No silver bullet, but a respectable arsenal. IEEE Comput. Graph. Appl., 22(6):2438, 2002.

15. Smart Surroundings, http://wwwes.cs.utwente.nl/smartsurroundings/. 

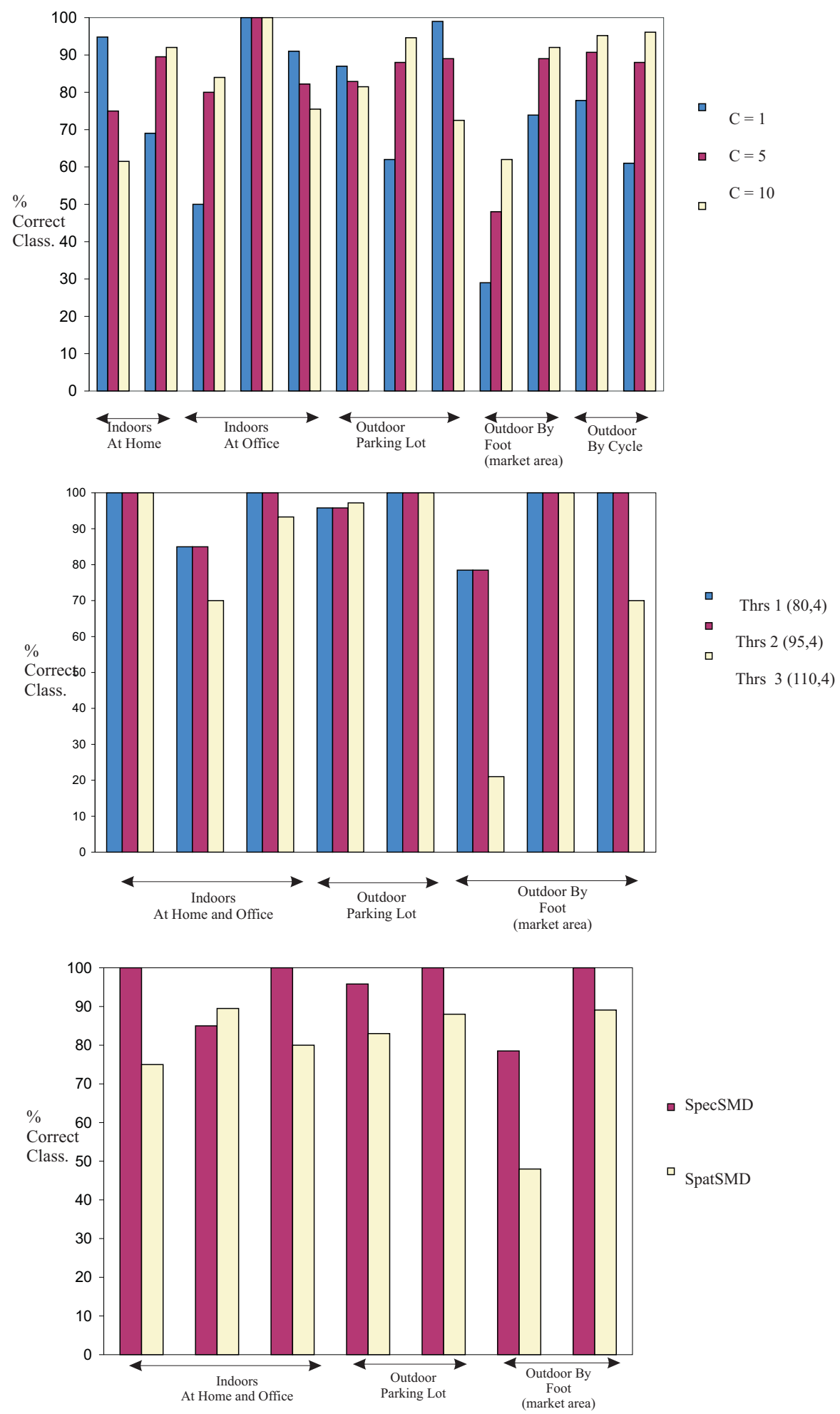

Fig. 6. (A). Accuracy results across different experimental set up for SpatSMDA Algorithm for various $\mathrm{C}$ values, with an average correct classification accuracy of $78.6 \%(\mathrm{C}=1), 86.7 \%(\mathrm{C}=5)$ and $87 \%(\mathrm{C}=10)$, (B). Accuracy results across different experimental set up for SpecSMDA Algorithm for various FWHM and counter threshold, Thrs 1: FWHM=80, Ctr Threshold =4, Thrs 2:FWHM= 95, Ctr Threshold $=4$ and Thrs 3:FWHM =110, Ctr Threshold =4, (C). Accuracy comparison between SpecSMD and SpatSMD, against same data sets. 